Supporting Information

\title{
Low Dimensional Platinum-Based Bimetallic Nanostructures for Advanced Catalysis
}

\author{
Qi Shao, Pengtang Wang, Ting Zhu, and Xiaoqing Huang*
}

College of Chemistry, Chemical Engineering and Materials Science, Soochow University, Suzhou, Jiangsu 215123, China. 
Table S1. Summary of activity and stability of low-D Pt based catalysts for ORR.

\begin{tabular}{|c|c|c|c|c|c|}
\hline Catalyst & Electrolyte & $\begin{array}{c}\text { Mass Activity at } \\
0.9 \mathrm{~V}\end{array}$ & $\begin{array}{c}\text { Specific } \\
\text { Activity at } 0.9 \\
\text { V }\end{array}$ & Stablity & Reference \\
\hline $\begin{array}{c}\text { Ultrafine Jagged } \\
\text { Platinum Nanowires }\end{array}$ & $0.1 \mathrm{M} \mathrm{HClO}_{4}$ & 13.6 $\mathrm{A} \mathrm{mg}^{-1}$ & $10.8 \mathrm{~mA} \mathrm{~cm}-2$ & $\begin{array}{l}\text { Mass activity: } \\
11.9 \mathrm{~A} \mathrm{mg}^{-1} \text { and } \\
\text { specific activity: } \\
0.98 \mathrm{~mA} \mathrm{~cm}^{-2} \\
\text { after } 6000 \text { cycles }\end{array}$ & $\begin{array}{c}\text { Science 2016, 354, } \\
1414-1419\end{array}$ \\
\hline PtCo Nanowires & $0.1 \mathrm{M} \mathrm{HClO}_{4}$ & $3.71 \mathrm{~A} \mathrm{mg}^{-1}$ & $7.12 \mathrm{~mA} \mathrm{~cm}^{-2}$ & $\begin{array}{l}\text { Mass activity: } \\
3.41 \mathrm{~A} \mathrm{mg}^{-1} \mathrm{Pt} \\
\text { after } 20000 \\
\text { cycles }\end{array}$ & $\begin{array}{l}\text { Nat. Commun. } \\
2016,7,11850\end{array}$ \\
\hline $\begin{array}{l}\text { PtNiCo Ultrathin } \\
\text { Nanowires }\end{array}$ & $0.1 \mathrm{M} \mathrm{HClO}_{4}$ & $4.20 \mathrm{~A} \mathrm{mg}^{-1}$ & $5.11 \mathrm{~mA} \mathrm{~cm}^{-2}$ & $\begin{array}{l}\text { Mass activity: } 3.5 \\
\mathrm{~A} \mathrm{mg}^{-1}{ }_{\mathrm{Pt}} \text { after } \\
30000 \text { cycles }\end{array}$ & $\begin{array}{c}\text { Sci. Adv. 2017, 3, } \\
\text { e1601705 }\end{array}$ \\
\hline $\begin{array}{c}\text { PtNiRh Trimetallic } \\
\text { Nanowires }\end{array}$ & $0.1 \mathrm{M} \mathrm{HClO}_{4}$ & $2.88 \mathrm{~A} \mathrm{mg}^{-1}$ & $2.71 \mathrm{~mA} \mathrm{~cm}-2$ & $\begin{array}{l}\text { Mass activity: } \\
2.51 \mathrm{~A} \mathrm{mg}^{-1} \text { after } \\
10000 \text { cycles }\end{array}$ & $\begin{array}{c}\text { J. Am. Chem. Soc. } \\
2018,140,16159- \\
16167\end{array}$ \\
\hline $\begin{array}{l}\text { Ultrathin Rh doped Pt } \\
\text { Nanowires }\end{array}$ & $0.1 \mathrm{M} \mathrm{HClO}_{4}$ & $1.41 \mathrm{~A} \mathrm{mg}^{-1}$ & $1.63 \mathrm{~mA} \mathrm{~cm}^{-2}$ & $\begin{array}{l}\text { Mass activity: } \\
1.28 \mathrm{~A} \mathrm{mg}^{-1} \text { after } \\
10000 \text { cycles }\end{array}$ & $\begin{array}{c}\text { J. Am. Chem. Soc. } \\
\text { 2017, 139, 8152- } \\
8159\end{array}$ \\
\hline Pd@Pt Nanowires & $0.1 \mathrm{M} \mathrm{HClO}_{4}$ & $0.17 \mathrm{~A} \mathrm{mg}_{\mathrm{P}_{\mathrm{Pt}+\mathrm{Pd}}{ }^{-1}}$ & $0.4 \mathrm{~mA} \mathrm{~cm}^{-2}$ & $\begin{array}{l}\text { Mass activity: } \\
0.323 \mathrm{~A} \mathrm{mg}_{\mathrm{P}+\mathrm{Pd}}{ }^{-1} \\
\text { and specific } \\
\text { activity: } 0.98 \mathrm{~mA} \\
\mathrm{~cm}^{-2} \text { after } 60000 \\
\text { cycles }\end{array}$ & $\begin{array}{c}\text { J. Am. Chem. Soc. } \\
\text { 2015, 137, 7862- } \\
7868\end{array}$ \\
\hline PtNiPd Nanowires & $0.1 \mathrm{M} \mathrm{HClO}_{4}$ & $1.93 \mathrm{~A} \mathrm{mg}^{-1}$ & $3.48 \mathrm{~mA} \mathrm{~cm}^{-2}$ & $\begin{array}{l}\text { Mass activity: } \\
1.78 \mathrm{~A} \mathrm{mg}^{-1} \text { after } \\
10000 \text { cycles }\end{array}$ & $\begin{array}{c}\text { Adv. Mater. 2017, } \\
29,1603774\end{array}$ \\
\hline $\begin{array}{l}\text { Zigzag-Like PtFe } \\
\text { Nanowires }\end{array}$ & $0.1 \mathrm{M} \mathrm{HClO}_{4}$ & $2.11 \mathrm{~A} \mathrm{mg}^{-1}$ & $4.34 \mathrm{~mA} \mathrm{~cm}^{-2}$ & $\begin{array}{l}\text { Mass activity: } \\
1.59 \mathrm{~A} \mathrm{mg}^{-1} \text { and } \\
\text { specific activity: } \\
3.18 \mathrm{~mA} \mathrm{~cm}^{-2} \\
\text { after } 50000 \\
\text { cycles }\end{array}$ & $\begin{array}{c}\text { Adv. Mater. 2018, } \\
\text { 30, } 1705515\end{array}$ \\
\hline $\mathrm{PtPb} / \mathrm{Pt}$ Nanoplate & $0.1 \mathrm{M} \mathrm{HClO}_{4}$ & $4.3 \mathrm{~A} \mathrm{mg}^{-1}$ & $7.8 \mathrm{~mA} \mathrm{~cm}^{-2}$ & $\begin{array}{l}\text { Mass activity: } \\
3.97 \mathrm{~A} \mathrm{mg}^{-1} \text { after } \\
50000 \text { cycles }\end{array}$ & $\begin{array}{c}\text { Science 2016, 354, } \\
1410-1414\end{array}$ \\
\hline PtPb Nanoplate & $0.1 \mathrm{M} \mathrm{HClO}_{4}$ & $0.84 \mathrm{~A} \mathrm{mg}^{-1}$ & $1.01 \mathrm{~mA} \mathrm{~cm}-2$ & $\begin{array}{c}\text { Mass activity: } \\
0.75 \mathrm{~A} \mathrm{mg}^{-1} \text { and } \\
\text { specific activity: } \\
0.92 \mathrm{~mA} \mathrm{~cm}^{-2}\end{array}$ & $\begin{array}{c}\text { Small 2018, 14, } \\
1702259\end{array}$ \\
\hline
\end{tabular}


after 50000

cycles

$\mathrm{Pd}_{3} \mathrm{~Pb} / \mathrm{Pd}$ Nanosheet

$0.1 \mathrm{M} \mathrm{KOH}$

$0.469 \mathrm{~A} \mathrm{mg}_{\mathrm{Pd}}{ }^{-1}$

$1.31 \mathrm{~mA}$

Mass activity:

Nano Lett. 2019 ,

$\mathrm{cm}_{\mathrm{Pd}}{ }^{-2}$

$0.43 \mathrm{~A} \mathrm{mg}_{\mathrm{Pd}^{-1}}$

192, 1336-1342

after 20000

cycles 
Table S2. Summary of activity and stability of low-D Pt based catalysts for HER.

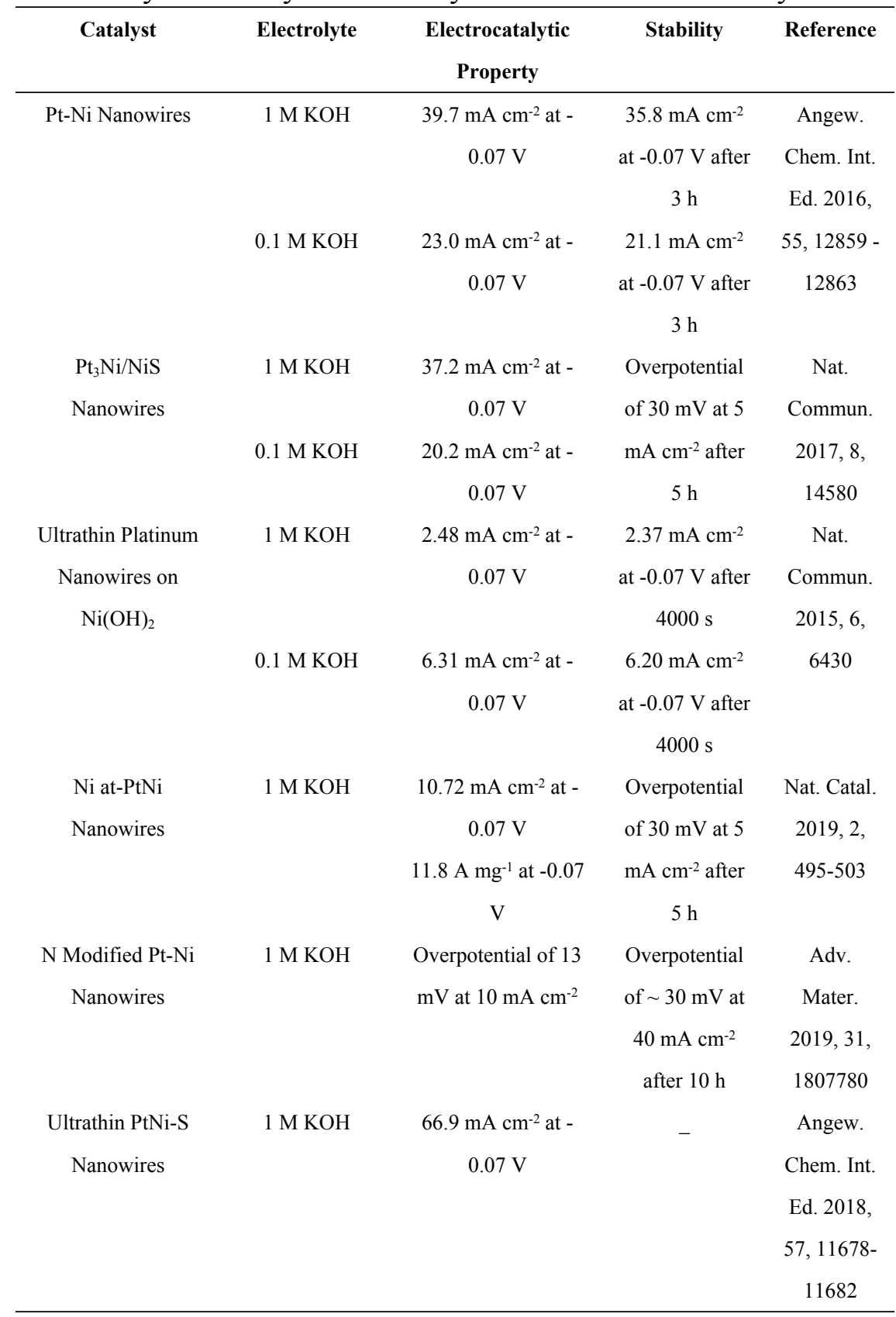

